
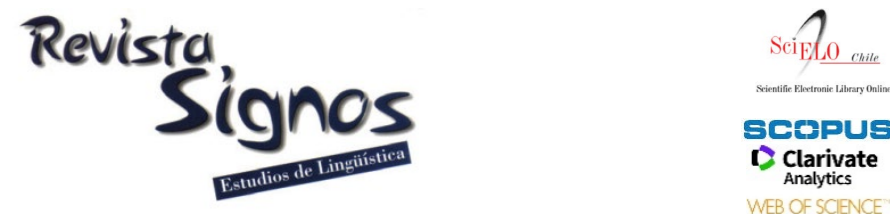

WEB OF SCIENCE

\title{
Las respuestas de los niños en discusiones argumentativas relacionadas con las reglas y normas de los padres
}

\section{Children's answers in argumentative discussions related to parental rules and norms}

\author{
Antonio Bova \\ Università Cattolica del SACro CuOre \\ ITALIA \\ antonio.bova@unicatt.it
}

Francesco Arcidiacono

Haute ECole PÉdagogique BEJUNE

SUIZA

Francesco.Arcidiacono@hep-bejune.ch

Recibido: 28-VII-2018 / Aceptado: 22-IV-2019

DOI: $10.4067 /$ S0718-09342020000100008

\section{Resumen}

Este estudio se propone investigar los tipos de respuestas de los niños de edades comprendidas entre 3 y 7 años en discusiones argumentativas relacionadas con las reglas y normas de los padres. El corpus de datos se compone de 132 discusiones argumentativas seleccionadas de 30 comidas grabadas en video de 10 familias suizas y italianas. Los datos se presentan mediante extractos discursivos de discusiones argumentativas y se analizan mediante el modelo ideal pragma-dialéctico de discusión crítica. Los hallazgos muestran que cuando los padres usan argumentos contextuales como los argumentos de calidad de comida, los niños reflejan los mismos tipos de argumentos. Por otro lado, cuando los padres utilizan argumentos más complejos, elaborados y sin contexto, los niños generalmente no usan ningún argumento, pero su respuesta es una expresión de dudas o una mera oposición sin proporcionar ningún argumento. En general, los resultados de este estudio indican que los tipos de respuestas de los niños están estrictamente conectadas con el tipo de argumento previamente utilizado por sus padres. Este aspecto es particularmente relevante en términos de las capacidades de los niños para participar en intercambios argumentativos y reaccionar de manera racional durante el enfrentamiento con los padres. Se necesita una investigación adicional en esta dirección para comprender mejor las potencialidades específicas del lenguaje en el proceso cotidiano de socialización dentro del contexto familiar.

Palabras Clave: Argumentación, familia, hora de comer, socialización del lenguaje, interacción padre-hijo. 


\begin{abstract}
This study seeks to investigate the types of responses by children aged between 3 and 7 years in argumentative discussions relating to parental rules and prescriptions. The data corpus is composed of 132 argumentative discussions selected from 30 video-recorded meals of 10 middle to upper-middle-class Swiss and Italian families. Data are presented through discursive excerpts of argumentative discussions and analysed by the pragmadialectical ideal model of critical discussion. The findings show that when parents advance context-bound arguments such as the arguments of quality (e.g., very good, salty, or not good) and quantity (e.g., too little, quite enough, or too much) of food, the arguments advanced by children mirror the same types of arguments previously used by parents. On the other hand, when parents advance more complex, elaborated, and context-unbound arguments such as the appeal to consistency's argument, the argument from authority and the argument from analogy, the children typically did not advance any argument, but their response is an expression of further doubt or a mere opposition without providing any argument. Overall, the results of this study indicate that the types of children's responses are strictly connected to the type of argument previously advanced by their parents. This aspect is particularly relevant in terms of children's capacities to engage in argumentative exchanges and to react in rational ways during the confrontation with the parents. Further research in this direction is needed in order to better understand specific potentialities of language in the everyday process of socialization within the family context.
\end{abstract}

Key Words: Argumentation, mealtime, family, language socialization, parent-child interaction.

\title{
INTRODUCCIÓN
}

La hora de comer representa una actividad crucial para investigar cómo los padres y los niños interactúan y argumentan, ya que es una de las pocas ocasiones durante el día que reúne a todos los miembros de la familia (Ochs, Pontecorvo \& Fasulo, 1996; Fiese, Foley \& Spagnola, 2006; Bova \& Arcidiacono, 2015). En el marco de la investigación de argumentación familiar (Pontecorvo \& Fasulo, 1999; Brumark, 2008; Arcidiacono \& Pontecorvo, 2009; Bova \& Arcidiacono, 2013a, 2013b, 2014a; Bova, 2015a, 2015b), este estudio se propone investigar los tipos de respuestas de niños de entre 3 y 7 años en discusiones durante las comidas relacionadas con las reglas de los padres. Nuestro objetivo es investigar las capacidades de los niños para participar en intercambios argumentativos y reaccionar de manera racional durante el enfrentamiento con sus padres. En particular, las siguientes dos preguntas de investigación guiarán este estudio: (1) En discusiones sobre reglas / normas parentales, ¿cuándo los niños usan argumentos para refutar los argumentos de sus padres? (2) Cuando los niños intentan refutar los argumentos de sus padres, ¿qué tipo de argumentos usan? Estas preguntas de investigación serán respondidas por medio de un análisis cualitativo de un corpus constituido por 132 discusiones argumentativas entre padres e hijos. 
El enfoque analítico para el análisis de las discusiones argumentativas entre padres e hijos se basa en el modelo ideal pragma-dialéctico de discusión crítica (van Eemeren \& Grootendorst, 2004). Este sistema propone una definición ideal de argumentación desarrollada de acuerdo con el estándar de razonabilidad: una discusión argumentativa comienza cuando el hablante da su punto de vista, y el oyente arroja dudas sobre ella, o directamente contrasta el punto de vista. En consecuencia, la confrontación es una condición necesaria para que se produzca una discusión argumentativa. Este modelo se ajusta particularmente a este estudio, y más generalmente, al estudio de las interacciones argumentativas que ocurren en contextos ordinarios, como las conversaciones familiares, porque proporciona criterios específicos para seleccionar e identificar las discusiones argumentativas y los argumentos utilizados por los participantes. Hasta ahora, el modelo pragma-dialéctico y la noción de discusión argumentativa de van Eemeren y Grootendorst (2004) no se han utilizado explícitamente en investigaciones centradas en el lenguaje de los niños. Según este modelo, la determinación analítica del discurso se logra interpretando cada uno de sus componentes desde la perspectiva de la resolución de una diferencia de opinión. Con respecto al examen de conversación en interacción, el uso de este modelo como una guía para la reconstrucción argumentativa tiene como objetivo producir una visión analítica de todos los componentes de un discurso, qué puntos están en discusión y cuáles argumentos explícitos e implícitos están avanzados. En este estudio, queremos ampliar el conocimiento existente sobre la argumentación familiar adoptando este modelo analítico, como una cuadrícula para el análisis, ya que proporciona los criterios para la selección de las discusiones argumentativas y para la identificación de los argumentos presentados por los padres y los niños.

En el presente documento, la Sección 2 presentará una discusión concisa de la literatura más relevante sobre interacciones argumentativas en familia. En la Sección 3, se describirá la metodología. Los resultados del análisis se discuten en la Sección 4, seguida de la Sección 5, que resume los principales hallazgos y comentarios sobre sus limitaciones y fortalezas.

\section{Interacciones argumentativas durante las comidas familiares}

Junto con una serie de estudios que han resaltado las ventajas cognitivas y educativas de remodelar las actividades de enseñanza y aprendizaje en términos de interacciones argumentativas (ver, por ejemplo, Muller Mirza \& Perret-Clermont, 2009; Arcidiacono \& Bova, 2017), la relevancia de la argumentación en el contexto familiar está emergiendo rápidamente en los estudios de argumentación. En particular, la estructura y los elementos lingüísticos que caracterizan las interacciones argumentativas entre padres e hijos pequeños han sido investigados por varios académicos. La interacción entre argumentos y contraargumentos es evidente en el marco de situaciones antagónicas entre padres e hijos (Arcidiacono \& Pontecorvo, 
2009; Bova \& Arcidiacono, 2013a, 2018). Goodwin (2006), al analizar una disputa entre un padre y su hijo, ha demostrado cómo las expresiones que se oponen a otra posición en un argumento se construyen con una orientación simultánea a la estructura detallada del enunciado anterior al oponerse y las trayectorias de acción futuras proyectadas por ese enunciado, que el enunciado actual intenta contrarrestar e interceptar. Examinando el análisis secuencial del uso de directivas en conversaciones entre padres y niños pequeños durante las comidas, M. H. Goodwin (2006) ha demostrado cómo las formas de argumentos construidos con posiciones recicladas difieren de manera importante de los argumentos en los que los niños participan al dar cuenta de su propio comportamiento con sus padres. Las situaciones en las que los niños eluden sus responsabilidades pueden llevar a una escalada de afirmaciones de autoridad a través de amenazas o la renuncia de un padre a la derrota. Por el contrario, cuando los padres persisten en seguir sus instrucciones, a menudo facilitados por situaciones en las que los niños y los padres se unen para mantener el contacto cara a cara entre sí, los niños aprenden a ser responsables de sus acciones.

En cuanto a la argumentación oral de los niños, la mayoría de los estudiosos coinciden con la afirmación de que la capacidad de comprender y producir argumentos emerge al principio del desarrollo (por ejemplo, Orsolini, 1993; Anderson, Chinn, Chang, Waggoner \& Yi, 1997; Stein \& Albro, 2001; Arcidiacono \& Bova, 2011; Alam \& Rosemberg, 2014; Migdalek, Rosemberg \& Santibáñez Yáñez, 2014a; Pauletto, Aronsson \& Arcidiacono, 2017). Dunn y Munn (1987) mostraron que a los 4 años los niños, en conversaciones con su madre, pueden justificar su posición argumentando sobre las consecuencias de sus acciones. A la edad de 5 años, los niños aprenden a oponerse hacia sus padres y se convierten en participantes verbales activos en conflictos familiares. Arcidiacono y Bova (2015) observaron que durante las conversaciones a la hora de la comida con sus padres, los niños hacen uso de sofisticadas habilidades argumentativas cuestionando las reglas impuestas por sus padres. Brumark (2008) mostró que los adolescentes de 12 a 14 años usan argumentos que duran más tiempo y requieren más argumentos para ser resueltos, mientras que los niños de 7-10 años usan argumentos más cortos que se refieren al contexto inmediato. Hester y Hester (2010) demostraron que los niños pueden usar tanto los recursos contextuales como los culturales para producir sus argumentos.

Esta discusión crítica de la literatura muestra que los estudios sobre las interacciones argumentativas entre los miembros de la familia durante la hora de la comida han prestado considerable atención a la investigación de las estrategias argumentativas adoptadas por los padres y las habilidades argumentativas de los niños pequeños. Este estudio tiene como objetivo proporcionar una contribución adicional a la investigación sobre la argumentación familiar: el propósito de este artículo es investigar los tipos de respuestas de niños de entre 3 y 7 años en discusiones argumentativas relacionadas con las reglas y normas de los padres. 


\section{Metodología}

\subsection{Corpus de datos}

El corpus de datos se compone de treinta comidas familiares separadas y grabadas en video (que constituyen unas veinte horas de datos de video), construidas a partir de dos conjuntos diferentes de datos, llamados subcorpus 1 y subcorpus 2. Todos los participantes son de habla italiana. La duración de las grabaciones varía de 20 a 40 minutos. El sub-corpus 1 consta de 15 comidas grabadas en video en cinco familias italianas con un alto nivel socioeconómico ${ }^{1}$ que viven en Roma. Los criterios adoptados en la selección de las familias italianas fueron los siguientes: la presencia de ambos padres y al menos dos hijos, de los cuales el menor es de edad preescolar ( 3 a 6 años). La mayoría de los padres en el momento de la recopilación de datos tenían alrededor de 30 años $(\mathrm{M}=37,40, \mathrm{DE}=3,06)$. Todas las familias en el sub-corpus 1 tuvieron dos hijos. El sub-corpus 2 consta de 15 comidas grabadas en video en cinco familias suizas con un alto nivel socioeconómico, todos residentes en el área de Lugano. Los criterios adoptados en la selección de las familias suizas reflejan los criterios adoptados en la creación del sub-corpus 1. En el momento de la recolección de datos, la mayoría de los padres tenían alrededor de 30 años $(\mathrm{M}=35.90$, $\mathrm{SD}=$ 1.91). Las familias tenían dos o tres hijos.

\subsection{Procedimientos de recopilación y transcripción de datos}

En una primera fase, dos investigadores adoptaron completamente todas las comidas familiares adoptando el sistema de transcripción CHAT (MacWhinney, 2000), con algunas modificaciones introducidas para mejorar la legibilidad. El nivel de acuerdo entre los dos investigadores, medido por el alfa de Cronbach, fue .82. Posteriormente, los investigadores revisaron junto con los miembros de la familia las transcripciones en su hogar. Este procedimiento permite pedir a los miembros de la familia que aclaren pasajes que no estaban claros para los investigadores debido a un bajo nivel de sonido de grabación y palabras y construcciones vagas. La información sobre el entorno físico de las comidas, es decir, una descripción de la cocina y de la mesa de comedor, también se hizo para cada comida familiar.

\subsection{Método analítico}

El enfoque analítico adoptado para el análisis es el modelo ideal pragma-dialéctico de discusión crítica (van Eemeren \& Grootendorst, 2004). Este sistema considera que los actos de habla argumentativos no se realizan en un vacío social, sino entre dos o más partes que están teniendo un desacuerdo e interactúan entre sí en un intento de resolver este desacuerdo. El modelo de una discusión crítica explica cuatro etapas que son necesarias para una resolución dialéctica de las diferencias de opinión (van Eemeren \& Grootendorst, 2004): 'Etapa de confrontación': se establece que hay una disputa. Un punto de vista es utlizado y cuestionado; 'Etapa de apertura': se toma la 
decisión de intentar resolver la disputa por medio de una discusión argumentativa regulada; una de las partes asume el papel de protagonista, lo que significa que está preparado para defender su punto de vista mediante la argumentación; la otra parte asume el papel de antagonista, lo que significa que está preparado para desafiar sistemáticamente al protagonista para defender su punto de vista; 'Etapa de argumentación': el protagonista defiende su punto de vista y el antagonista le provoca una mayor argumentación si tiene más dudas; 'Etapa final': en la etapa de conclusión, se establece si la disputa se ha resuelto debido al punto de vista o si la duda sobre el punto de vista se ha retractado.

En el presente estudio, el modelo ideal de discusión crítica se asume como una cuadrícula para el análisis, ya que proporciona los criterios para la selección de las discusiones argumentativas y para la identificación de los argumentos presentados por los padres y los niños.

\subsection{Definición de situación argumentativa y selección de los argumentos}

El análisis presentado en este documento se centrará en el estudio de movimientos argumentativos analíticamente relevantes, es decir, "those speech acts that (at least potentially) play a role in the process of resolving a difference of opinión" (nuestra traducción: "aquellos actos de habla que (al menos potencialmente) desempeñan un papel en el proceso de resolver una diferencia de opinión") (van Eemeren \& Grootendorst, 2004: 73). En particular, para el presente estudio, solo las discusiones que cumplen los tres criterios siguientes fueron seleccionadas para el análisis: 1) una diferencia de opinión entre padres e hijos sobre un problema relacionado con las reglas y normas de los padres se hace evidente en el discurso: ‘¿Puede el niño usar la goma para borrar el dibujo?; 2) al menos un punto de vista utlizado por uno de los dos padres es cuestionado verbalmente por uno o más niños, o viceversa: (NIÑO) 'Sí, quiero intentar' - (PADRE) 'No, no puedes'; 3) al menos uno de los dos padres presenta al menos un argumento a favor o en contra del punto de vista que se cuestiona: que el caucho es para el tablero de dibujo y no se puede usar en otras cosas.

Para identificar los tipos de argumentos presentados por padres e hijos, el análisis se centra en la tercera etapa del modelo de una discusión crítica, a saber, la etapa de argumentación. Como lo expresan van Eemeren y Grootendorst (1992: 138):

"The dialectical objective of the parties is to test the acceptability of the standpoints that have shaped the difference of opinion" (nuestra traducción: "El objetivo dialéctico de las partes es probar la aceptabilidad de los puntos de vista que han configurado la diferencia de opinión"). 


\subsection{Tipos de argumentos presentados por padres e hijos}

Las categorías de argumentos identificadas en el presente estudio se basan en estudios previos de académicos de la argumentación y ya se han discutido en la literatura pertinente (por ejemplo, van Eemeren \& Grootendorst, 1992; Walton, Reed \& Macagno, 2008). A continuación, una breve definición de cada tipo de argumento identificado en el corpus.

El argumento de la calidad puede referirse a una propiedad, positiva o negativa, de algo, o a un determinado comportamiento de alguien, mientras que el argumento de la cantidad puede referirse a la cantidad y/o el tamaño de algo, o a un determinado comportamiento de alguien (por ejemplo, ver Bova \& Arcidiacono, 2014a). En nuestro corpus, los argumentos de calidad y cantidad se usan típicamente en discusiones relacionadas con la comida servida y con el comportamiento de los niños. Esto no es para nada sorprendente, ya que las conversaciones a la hora de comer suelen ser prácticas de alimentación. Los otros tipos de argumentos propuestos por los padres con sus hijos, es decir, los argumentos de la autoridad, el argumento del pedido de coherencia y el argumento de la analogía, se utilizan en discusiones relacionadas con una gama más amplia de temas tales como la enseñanza del comportamiento correcto en situaciones dentro y fuera del contexto familiar, por ejemplo, en el contexto escolar con profesores y compañeros.

El argumento de la autoridad fue otro tipo de argumento utilizado en algunos casos por los padres en discusiones argumentativas relacionadas con las reglas y normas de los padres con sus hijos. Aquí, nos referemos a la definición de argumento de la autoridad como la noción de autoridad deóntica elaborada por Walton (1997: 88):

"The deontic type of authority is a right to exercise command or to influence, especially concerning rulings on what should be done in certain types of situations, based on an invested office, or an official or recognized position of power" (nuestra traducción: "El tipo de autoridad deóntica es un derecho a ejercer el mando o influir, especialmente en lo que respecta a las decisiones sobre lo que se debe hacer en ciertos tipos de situaciones, sobre la base de una posición de poder oficial o reconocida").

En particular, los criterios para seleccionar los argumentos de la autoridad utilizada por los padres con sus hijos se pueden describir a través de la siguiente declaración: La persona X dijo/hizo Y, por lo tanto Y debe ser correcta/aceptada (para un estudio detallado de este tipo de argumento utilizado por los niños, véase también Bova, 2015a). 
El argumento del pedido a la coherencia se puede describir a través de la siguiente pregunta: Si lo ha afirmado explícita o implícitamente en el pasado, ¿por qué no lo está manteniendo ahora? (por ejemplo, vea Bova \& Arcidiacono, 2014b).

En cuanto al argumento de la analogía, en el presente estudio nos referemos a la definición dada por Walton et al. (2008). El razonamiento detrás de este argumento es el siguiente: premisa mayor: en general, el caso C1 es similar al caso C2 (por ejemplo, 'el clima en enero es similar al clima en diciembre'); premisa ninor: la proposición A es verdadera en el caso C1 (por ejemplo, 'en diciembre llovió todos los días'); conclusión: la proposición A es verdadera en el caso C2 (por ejemplo, 'en enero lloverá todos los días').

\section{Análisis y resultados}

Todo el corpus estaba compuesto por $\mathrm{N}=132$ discusiones argumentativas entre padres e hijos relacionadas con las reglas y normas de los padres. En el corpus, los padres presentaron al menos un argumento (en varios casos más de un solo argumento) en apoyo de sus reglas y normas en $\mathrm{N}=125$ instancias (95\%) para un número total de $\mathrm{N}=186$ argumentos. Los niños utlizaron al menos un argumento (en algunos casos más de un solo argumento) para refutar las reglas y normas parentales en $\mathrm{N}=48$ casos $(36 \%)$, para un número total de $\mathrm{N}=58$ argumentos.

Los argumentos dirigidos por los padres a sus hijos se pueden atribuir a cinco categorías argumentativas principales: calidad $(\mathrm{N}=79,42 \%)$, cantidad $(\mathrm{N}=52,28 \%)$, autoridad $(\mathrm{N}=31 ; 17 \%)$, pedido de coherencia $(\mathrm{N}=15 ; 8 \%)$ y la analogía $(\mathrm{N}=9$; $5 \%$ ). En el corpus, no observé diferencias significativas en los tipos de argumentos utilizados por las madres y los padres, con la excepción de los argumentos de la autoridad que fueron utilizados con mayor frecuencia por los padres que por las madres. Los argumentos expuestos por los niños en reacción a los argumentos de sus padres son, casi exclusivamente, argumentos de calidad $(\mathrm{N}=25$, aproximadamente $43 \%$ ) y cantidad $(\mathrm{N}=30,52 \%$ ). Solo en tres casos los niños presentaron un tipo de argumento diferente, es decir, el argumento de la autoridad en dos instancias y el argumento del pedido a la coherencia en un caso. A continuación se presenta una vista sintética de los argumentos presentados por los padres y de los tipos de respuestas y argumentos de los niños. En las siguientes secciones se incluirán extractos sobre las estrategias argumentativas utilizadas por los padres y los argumentos relacionados utilizados por los niños.

\subsection{Argumentos de calidad y cantidad}

En su mayoría, en las discusiones argumentativas relacionadas con las reglas y normas de los padres, los padres utilizaron argumentos de calidad (por ejemplo, nutritivos, sabrosos, hermosos, demasiado salados, duros, amables) $(\mathrm{N}=79 ; 42 \%)$ y 
cantidad (por ejemplo, demasiado, demasiado poco) $(\mathrm{N}=52 ; 28 \%$ ). En las 79 instancias en que los padres presentaron un argumento de calidad, los tipos de respuestas de los niños fueron los siguientes: aceptación inmediata de la argumentación de los padres en 6 casos (8\%), expresión de otras dudas en 17 casos (22\%), oposición sin proporcionar argumentos en 24 instancias (30\%) y en 32 instancias utlizaron un argumento (40\%). Los argumentos de los niños fueron en gran parte argumentos de calidad (28 instancias) y en algunos casos argumentos de cantidad (4 instancias). En las 52 instancias en que los padres presentaron un argumento de cantidad, los tipos de respuestas de los niños fueron los siguientes: aceptación inmediata de la argumentación de los padres en 6 casos $(12 \%)$, expresión de mayor duda en 11 casos (21\%), oposición sin proporcionando argumentos en 11 instancias (21\%), y en 24 casos utlizaron un argumento (46\%). Los argumentos de los niños fueron, en gran parte, argumentos de cantidad (21 instancias), en 2 casos, argumentos de calidad y, en 1 instancia, un argumento de pedido a la coherencia.

Los siguientes ejemplos son ilustraciones de cómo estos dos tipos de argumentos son utilizados por los padres y cómo los niños reaccionan a través de argumentos (apropiados). En los ejemplos uno y dos, las madres presentan un argumento de calidad y cantidad, respectivamente, para convencer a los niños a comer. Los niños, a su vez, presentan argumentos de calidad y cantidad para refutar los puntos de vista de la madre.

Extracto 1. Familia suiza. Participantes: padre (DAD, 35 años), madre (MOM, 33 años), Matteo (MAT, 7 años 3 meses), Carlo (CAR, 4 años 8 meses), Alessia (ALE, 3 años 4 meses). Todos los miembros de la familia están sentados en la mesa. La cena casi ha terminado. Los padres hablan entre ellos sobre la comida, mientras sus hijos terminan de comer. En la línea 1, la madre le pregunta al padre si él también piensa que la comida que se sirvió durante la comida fue buena. El padre está de acuerdo con la madre (línea 2: ¡muy rica!). Inmediatamente después, la madre expresa su preocupación porque, dice, a su hijo de 7 años, Matteo, no ha comido nada durante la comida (línea 3). Este comportamiento contrasta con la excelente calidad de los alimentos reconocidos por ambos padres al comienzo de la secuencia.

1. *MOM: rica ((la comida)) esta noche, ¿verdad? [hablando con DAD]

2. *DAD: muy rica! \%act: $M O M$ mira hacia $M A T$

3. *MOM: mi madre! Matteo no comió nada esta noche [hablando con DAD]

4. *MOM:Matteo, tienes que comer albóndigas.

5. *MAT: no:: no las quiero ((albóndigas))

6. *MOM: jmira qué crujientes son! ((albóndigas))

7. *MAT: no:: ¡son duras! ((albóndigas)) \%act: MOM prueba las albóndigas

8. *MOM: sí, no están realmente ricas 
\%act: MOM mira hacia DAD

\%pau: 1.5 . sec

9. *MOM: ¿quieres un pequeño escalope?

10. *MAT: sí::! ((risa))

Dentro de este extracto, nos enfocaremos en la diferencia de opinión entre la madre y su hijo en las líneas 4-7. De hecho, la madre, en la línea 4, le dice a Matteo que debe comer las albóndigas, pero el niño inmediatamente no está de acuerdo con su madre (línea 5: no, no las quiero). En términos argumentativos, este intercambio representa una etapa de confrontación, ya que la madre y Matteo muestran dos puntos de vista opuestos: por un lado, la madre quiere que Matteo coma las albóndigas, mientras que, por el contrario, Matteo no quiere comerlas. En este punto, ella presenta una discusión apoyando su punto de vista. El argumento de la madre en la línea 6 se basa en la calidad de las albóndigas y, en particular, apunta enfatizando el buen gusto de la comida. Matteo responde que las albóndigas no están crujientes, sino que están duras (línea 7).

Los argumentos presentados por la madre y su hijo tienen como objetivo mostrar una propiedad específica de la comida servida durante la comida. Lo que distingue la argumentación de la madre y el niño es un juicio opuesto con respecto a la calidad de los alimentos. Mientras que el argumento de la madre apunta a subrayar una propiedad positiva de las albóndigas, el uso del adjetivo 'difícil' por Matteo indica a la madre una propiedad negativa de las albóndigas. En este caso, el argumento de calidad presentado por el niño es efectivo para convencer a su madre de que pruebe las albóndigas que ella misma ha preparado. Después de haber probado la albóndiga ella misma, de hecho, ella está de acuerdo con su hijo en que las albóndigas no son buenas (línea 8).

Extracto 2. Familia italiana. Participantes: padre (DAD, 41 años), madre (MOM, 38 años), Fabio (FAB, 7 años 2 meses), Luisa (LUI, 3 años 10 meses). Todos los miembros de la familia están sentados en la mesa. La cena comenzó hace quince minutos. Todos los miembros de la familia están comiendo el plato principal, es decir, el risotto. El extracto comienza cuando Fabio le dice a su madre que no quiere comer más risotto (línea 1: es suficiente, no quiero más).

\%sit: $\quad$ FAB está comiendo el risotto

1. $* \mathrm{FAB}:$ suficiente, no quiero más (risotto)

\%act: FAB deja de comer el risotto

2. *MOM: vamos, no has comido suficiente todavía

3. $* \mathrm{FAB}$ : pero si comí un kilo ((de risotto))

4. *MOM: vamos, todavía no has terminado

5. *FAB: no:: no::

\%act: FAB se levanta de la mesa y va hacia otra habitación 
En este ejemplo, la madre explícitamente no está de acuerdo con la declaración de su hijo (línea 1: eso es suficiente, no quiero más risotto), e inmediatamente presenta un argumento de cantidad para convencer a Giovanni de que coma un poco más. En la línea 3, Fabio le dice a su madre que ya ha comido un kilo de risotto. En este caso, podemos suponer razonablemente que según el niño, un kilo de risotto representa una gran cantidad. En consecuencia, le está diciendo a su madre que la cantidad de arroz que ha comido hasta ese momento es más que la cantidad correcta. Sin embargo, a diferencia del ejemplo anterior donde el argumento de la calidad fue efectivo para convencer a la madre de cambiar su opinión, el argumento presentado por el niño aquí no produce un resultado similar. La madre, de hecho, todavía quiere que Fabio termine de comer el risotto (línea 4: vamos: todavía no has terminado). Sin embargo, a pesar del esfuerzo argumentativo de la madre, Fabio todavía no está de acuerdo con ella y abandona la mesa (línea 5: no :: no ::). La secuencia argumentativa no encuentra una conclusión ni un compromiso entre los dos participantes. El retiro como posibilidad de cierre del intercambio verbal alrededor del risotto se puede considerar, en este caso, el signo de que uno de los participantes, es decir, el niño, se molestó demasiado para continuar la discusión.

En conclusión, en esta sección hemos visto que los argumentos de calidad y cantidad son los tipos de argumentos más frecuentes presentados por padres e hijos durante sus debates argumentativos durante las comidas. En particular, estos dos tipos de argumentos son utilizados en la mayoría de los casos por padres e hijos para resaltar una característica específica (positiva o negativa) de los alimentos. Además, hemos visto que los argumentos de calidad y cantidad fueron en su mayoría utlizados por los niños en reacción a los mismos tipos de argumentos previamente presentados por sus padres. De hecho, en el corpus cuando el padre presentó un argumento de calidad o un argumento de cantidad, el argumento del niño típicamente reflejaba el mismo tipo de argumento previamente propuesto por sus padres.

\subsection{Argumento de la autoridad}

El conjunto de datos en el presente estudio se compone de $\mathrm{N}=31$ (17\%) argumentos de la autoridad que cumplen los criterios descritos anteriormente. En los 31 casos en que los padres presentaron un argumento de autoridad, los tipos de respuestas de los niños fueron los siguientes: aceptación inmediata de la argumentación de los padres en 3 casos (10\%), expresión de otras dudas en 11 casos (36\%), oposición sin proporcionando argumentos en 15 instancias (48\%), y solo en 2 casos utlizaron un argumento $(6 \%)$. En ambos casos, los argumentos presentados por los niños eran argumentos de la autoridad. Además, en comparación con los argumentos de calidad y cantidad, que en la mayoría de los casos utlizaron durante las discusiones relacionadas con los alimentos, en el corpus este tipo de argumento fue utilizado en la mayoría de los casos por los padres en discusiones donde su propósito era enseñar el comportamiento correcto en situaciones sociales dentro y fuera del 
contexto familiar para sus hijos. El siguiente diálogo entre una madre y su hijo de 4 años, Domenico, ofrece un ejemplo de este tipo de argumento.

Extracto 3. Familia suiza. Participantes: padre (DAD, 36 años), madre (MOM, 34 años), Stefano (STE, 8 años y 5 meses), Domenico (DOC, 4 años y 6 meses). A excepción de DAD, que está en la cocina, todos los miembros de la familia están sentados en la mesa en el comedor. La cena está casi terminando. La madre les pregunta a los niños si todavía quieren comer un poco más de comida (línea 1), pero los niños ya están concentrados en otras actividades, como jugar con juguetes y otros objetos. En esta fase de la comida, ha sido frecuente observar, en el corpus, discusiones en las que padres e hijos negocian las actividades posteriores a la cena, por ejemplo, cuánto tiempo mira televisión, si juega con tal o cual objeto, o en qué momento dormir. De hecho, las comidas no solo están orientadas a la alimentación, sino que representan momentos en los que todas las actividades diarias que involucran a los miembros de la familia, especialmente a los niños, pueden tenerse en cuenta y debatirse.

1. *MOM: Stefano y Domenico, ¿aún queréis comer algo?

2. *DOC: ¿Puedo tomar la goma para borrar el dibujo? \%act: $\quad$ MOM dice que no, moviendo un dedo

3. *MOM: no Domenico

$\rightarrow$ *MOM: no!

$\rightarrow *$ MOM: esa goma es para la pizarra,

$\rightarrow$ *MOM: y no puedes usarlo para otras cosas

[...]

8. *DOC: pero::

9. *MOM: ¿Quépasa?

10. *DOC: pero quiero probarlo

11. *MOM: no, cariño

12.*DOC: $\quad$ no::

13. *MOM: no cariño, créeme porque sé de lo que estoy hablando

$\rightarrow * \mathrm{MOM}:$ algunas veces, puedes intentarlo

$\rightarrow * \mathrm{MOM}:$ otras veces no puedes intentarlo, debes confiar en lo que tus padres te digan

14. *DOC: pero quiero borrarlo ((el dibujo))

15. *MOM: entonces espera que te dé la goma correcta

16. ${ }^{*}$ DOC: vale

En el extracto, el foco está en la discusión entre la madre y Domenico (línea 2, y de la línea 8 a la línea 16). La secuencia comienza cuando Domenico le dice a su madre que quiere tomar una goma para borrar un dibujo en la pizarra. La madre no está de acuerdo con Domenico y, en la línea 3, le revela a su hijo en qué se basa su opinión (esa goma es para el tablero de dibujo y no puedes usarla en otras cosas). Domenico 
no está convencido por el argumento de su madre (línea 8: pero ::) y, en la línea 10, él responde que quiere intentar usar el borrador (pero quiero probarlo). En la línea 13, la madre usa un argumento que ya no está relacionado con las propiedades del borrador, pero establece una regla general que el niño debe seguir en situaciones similares. En este caso, el argumento de la madre es eficaz para convencer al niño de no usar la goma 'para el tablero de dibujo'. De hecho, Domenico acepta usar la 'goma derecha' que le dará su madre (línea 16). La mitigación utilizada por la madre en la línea 13 (a veces, puedes intentar) y la concesión en la línea 15 (espera hasta que te dé...) pueden considerarse formas de alinear su posición con el hijo debido a la discusión ofrecido por el niño en la línea 14 (pero quiero borrarlo): en este sentido, el esfuerzo de la madre de recontextualizar el reclamo puede verse como un buen compromiso entre el llamado a la autoridad y la opinión de los expertos y el razonabilidad del deseo y la intención del niño.

\subsection{Argumento del pedido a la coherencia}

El conjunto de datos en el presente estudio se compone de $\mathrm{N}=15(8 \%)$ argumentos del pedido a la coherencia. En las 15 instancias en que los padres presentaron un argumento del pedido a la coherencia, los tipos de respuestas de los niños fueron los siguientes: no aceptación inmediata de la argumentación de los padres, expresión de mayor duda en 6 casos (40\%), oposición sin proporcionar argumentos en 9 casos (60\%). El pedido a la coherencia, en la mayoría de los casos utilizados por los padres para enseñar a sus hijos el comportamiento correcto en situaciones sociales dentro y fuera del contexto familiar, parece ser un argumento bastante complejo, quizás demasiado complejo, para los niños, ya que en caso de que hayan utlizado un argumento en respuesta a los argumentos de sus padres. El siguiente ejemplo es una ilustración de este tipo de argumento.

Extracto 4. Familia suiza. Participantes: padre (DAD, 38 años), madre (MOM, 36 años), Stefano (STE, 7 años), Laura (LAU, 4 años 5 meses), Elisa (3 años 2 meses). Todos los miembros de la familia están sentados en la mesa. Los protagonistas del diálogo son una madre y su hijo, Stefano, de 7 años. Todos los miembros de la familia están comiendo el plato principal. En este momento de la conversación, el enfoque de los padres no está en la comida: están hablando del comportamiento escolar de uno de sus hijos. En la línea 1, la madre envía un cumplido a su hijo de 7 años: Stefano, fuiste muy bueno ayer. Con estas palabras, muestra su intención de iniciar una conversación con su hijo: sin embargo, Stefano parece desconcertado, porque no sabe la razón por la que, según su madre, ayer fue muy bueno (línea 2: ¿por qué?). En la línea 3, la madre revela el motivo por el cual se basa su cumplido a su hijo: ella dice que la tía Daniela le dijo que ayer fue muy bueno porque hizo toda la tarea escolar. En este punto de la secuencia, la madre presenta una frase que revela la consecuencia lógica del comportamiento del niño: ella quiere que Stefano vaya de nuevo a la casa de Daniela porque el día anterior él fue muy bueno. 
1. *MOM: Stefano, ayer fuiste muy bueno

2. *STE: ¿Por qué?

3. *MOM: ¿Por qué?

$\rightarrow$ *MOM: la tía Daniela me dijo que ayer fuiste muy bueno

$\rightarrow *$ MOM: biciste todos los deberes

$\rightarrow *$ MOM: entonces mañana vuelves donde la tía Daniela para hacer los deberes, ¿de acuerdo?

4. *STE: no :: no quiero

5. *MOM: vamos, Stefano

$\rightarrow *$ MOM: pero ayer estuviste alli toda la tarde

$\rightarrow$ *MOM: $i y$ hoy has dicho que te has divertido mucho!

6. *STE: $\quad m b m::((S T E$ tiene una expresión perpleja))

7. *MOM: vale, entonces mañana te llevo donde la tía Daniela

\%act: STE asiente con la cabeza y muestra que está de acuerdo con MOM

El razonamiento utilizado por la madre para justificar el hecho de que Stefano tiene que ir de nuevo a la casa de la tía Daniela se basa en la forma lógica 'como X, entonces $Y^{\prime}$ (dada la consistencia del primer elemento, el segundo elemento se justifica). Como primera reacción, Stefano no está de acuerdo con la propuesta de la madre (línea 4: no :: no quiero), desaprobando la lógica de la madre y expresando su sentimiento personal. Aquí, la madre sigue una estrategia interesante, ya que presenta dos argumentos de coordinación en la línea 5: ipero ayer estuviste allí toda la tarde y hoy dijiste que te divertías tanto!. Al referirse a una acción que hizo Stefano en el pasado (ayer estuviste allí toda la tarde) y enfatizabas lo bueno que era ese evento (hacer los deberes con la casa de la tía Daniela) para él (jhoy dijiste que te divertías tanto!), la madre intenta para mostrarle a Stefano que su comportamiento actual debe ser consistente con el del pasado. En este caso, los argumentos de coordinación presentados por la madre parecen ser efectivos para convencer a su hijo de cambiar su opinión (STE asiente con la cabeza a su madre para decir que está de acuerdo con ella), o, al menos, para aceptar el propuesta de la madre

En este ejemplo, podemos observar que al mantener su razonamiento argumentativo, la madre usó 'pero' en la línea 5. Esta elección probablemente se deba al hecho de que quiere subrayar la contradicción entre el comportamiento del hijo anterior (el tiempo pasado en el casa de la tía) y su reacción no coherente (no quiere volver) a la propuesta de la madre. Como ya dijo Schiffrin (1987), el efecto del marcador 'pero' puede reforzarse aún más a través de la conjunción y. Esto es exactamente lo que sucede en esta discusión, donde la madre después de usar el marcador 'pero' (pero ayer estuviste allí toda la tarde) refuerza su argumento al agregar la conjunción y (y hoy dijiste que tenías Muy divertido). Finalmente, en la etapa final de la secuencia, la madre hace explícita la lógica de su proceso de razonamiento, 
diciendo: 'así que mañana te llevaré a la tía Daniela' (línea 7), como consecuencia de la discusión usada desde el principio en línea 3.

\subsection{Argumentos de la analogía}

El conjunto de datos en el presente estudio se compone de $\mathrm{N}=9(5 \%)$ argumentos de analogía que cumplen los criterios descritos anteriormente. En los 9 casos en que los padres presentaron un argumento de analogía, los tipos de respuestas de los niños fueron los siguientes: no aceptación inmediata de la argumentación de los padres, expresión de otras dudas en 3 casos (33\%), oposición sin proporcionar argumentos en 6 casos (67\%). De manera similar a lo que hemos observado para el argumento de la autoridad y el argumento del pedido a la coherencia, este tipo de argumento fue utilizado casi exclusivamente por los padres en discusiones relacionadas con la enseñanza del comportamiento correcto en situaciones sociales dentro y fuera del contexto familiar para sus hijos. Además, como hemos visto con el argumento del pedido a la coherencia, en cualquier caso los niños han presentado un argumento para refutar el argumento adelantado por sus padres. El siguiente diálogo entre una madre y su hijo de 7 años, Francesco, ofrece un ejemplo de este tipo de argumento.

Extracto 5. Familia italiana. Participantes: padre (DAD, 42 años), madre (MOM, 40 años), Francesco (FRA, 7 años y 2 meses), Leonardo (LEO, 3 años y 9 meses). Todos los niños están sentados a la mesa. MOM está parada y está sirviendo la cena. DAD está sentado en el sofá viendo la televisión. Al comienzo de este extracto, la madre está sirviendo la comida, mientras que el padre todavía está sentado en el sofá viendo la televisión. Le pide al padre que se siente a la mesa y disfrute de la comida, ya que la comida está lista (línea 1: ven, la cena está lista). Este evento, es decir, la madre que anuncia el comienzo de la comida, representa un punto de partida típico para este tipo de actividad. En el extracto, enfocaremos el análisis sobre la diferencia de opinión entre la madre y su hijo, Francesco, sobre un tema relacionado con el contexto escolar (de la línea 3 a la línea 7).

1. *FRA: madre

2. *MOM: ¿Qué Francesco?

3. *FRA: según yo, la maestra Laura ((la profesora de matemáticas)) nos dará muchos deberes para las vacaciones ((refiriéndose a las vacaciones de Navidad))

4. *MOM: no:: no:

$\rightarrow *$ MOM: no lo creo

5. *FRA: sí, en cambio!

6. *MOM: no :: en mi opinión no.

$\rightarrow$ *MOM: si la maestra María ((la profesora de italiano)) no lo hizo, ni siquiera lo hará la maestra Laura

7. *FRA: esperemos! ((risa)) 
\%sit: $\quad$ MOM sonríe también

\%sit: $\quad$ MOM está sirviendo la comida a FRA

Francesco en la línea 3 usa un punto de vista: le dice a su madre que cree que la maestra de matemáticas, es decir, la maestra Laura, se los dará, esto significa no solo para él, sino para todos los alumnos de su clase, mucha tarea para hacer durante las vacaciones de Navidad. La madre no está de acuerdo con su hijo (línea 4: no :: no: no lo creo). Francesco, a su vez, decide estar en desacuerdo con su madre (línea 5: Sí, quiero), pero no ofrece ningún argumento en apoyo de su punto de vista. Para contrarrestar esto, la madre presenta un argumento de analogía para convencer a Francesco de que cambie su opinión. En la línea 6, de hecho, le dice a su hijo que si la maestra de italiano no les da deberes para hacer durante las vacaciones de Navidad, tampoco lo hará la maestra de matemáticas. El razonamiento detrás del argumento de la madre se puede inferir de la siguiente manera: debido a que las dos maestras comparten algunas similitudes, es decir, ambas son profesoras de la misma clase, se comportarán de manera similar. En este caso, el argumento presentado por la madre parece ser efectivo para convencer a su hijo de que cambie su opinión. Él no continúa defendiendo su punto de vista inicial (línea 7: jasí lo espero!) y concluyen la conversación sonriendo.

\section{DISCUSIÓN Y CONCLUSIÓN}

Este estudio tiene la intención de proporcionar una contribución al estudio de la discusión argumentativa en familias con niños pequeños. Nos hemos centrado especialmente en los tipos de respuestas de niños de edades comprendidas entre 3 y 7 años en discusiones argumentativas relacionadas con las reglas y normas de los padres. Como ya observó Blum-Kulka (1997) en su estudio transcultural sobre las conversaciones familiares, las discusiones argumentativas no están principalmente dirigidas a resolver conflictos verbales entre los miembros de la familia, pero esencialmente parecen ser un instrumento que permite a los padres transmitir, y a los niños aprender, valores y modelos sobre cómo comportarse de una manera culturalmente apropiada. Los propósitos por los cuales los padres pueden participar en una discusión argumentativa con sus hijos pueden ser diversos. A veces, necesitan presentar argumentos para justificar su punto de vista acerca de un cierto comportamiento o un cierto pensamiento que no es aceptado por sus hijos. Otras veces, los padres presentan argumentos para enseñarles a sus hijos cierto comportamiento correcto, por ejemplo, los modales correctos en la mesa, mientras que en otras ocasiones los padres presentan argumentos con el objetivo de involucrar a sus hijos en una nueva discusión, comenzando así un común razonamiento junto con ellos. Aunque hay muchos propósitos diferentes para los cuales los padres pueden entablar una discusión argumentativa con sus hijos, hay un elemento común a todas 
las discusiones argumentativas en las que participan: no hay argumentos sin argumentos que respalden un cierto punto de vista.

En línea con estudios precedentes (Pontecorvo \& Fasulo, 1999; Pontecorvo \& Pirchio, 2000; Goetz, 2010), los resultados de este estudio indican que los padres presentaron una mayor número de argumentos $(\mathrm{N}=186$ frente a $\mathrm{N}=58$ ) y utilizaron diferentes tipos de argumentos en comparación con sus hijos. Sin embargo, desde un punto de vista argumentativo, los resultados de este estudio sacan a la luz otro aspecto interesante: en sus elecciones argumentativas, los padres y los hijos se influyen mutuamente. Las interacciones argumentativas de la familia deben considerarse como un proceso bidireccional de aprendizaje mutuo en el que los padres influyen a los niños y son influenciados simultáneamente por ellos (Pontecorvo, Fasulo \& Sterponi, 2001; Arcidiacono \& Pontecorvo, 2009). De hecho, al participar en discusiones argumentativas, los padres aceptan el compromiso de aclarar a sus hijos las razones en las que se basan sus reglas, valores y normas, mientras que los niños pueden ser más conscientes de ser participantes activos en su propia familia. En consecuencia, por las razones mencionadas anteriormente, los padres desempeñan el papel de educadores durante las discusiones argumentativas y los niños desempeñan el papel no menos importante de los aprendices activos.

En ambos casos (padres e hijos), el uso predominante de argumentos relativos a los conceptos de cantidad y calidad aparece como la forma privilegiada de convencer a la otra parte. Cuando los niños presentaron argumentos utlizados para oponerse a la argumentación de sus padres, en su mayoría utilizaron el mismo tipo de argumento utilizado anteriormente por sus padres. Los argumentos de calidad y cantidad fueron en su mayoría utlizados por los niños en reacción a los mismos tipos de argumentos previamente presentados por sus padres. De hecho, en el corpus observamos que cuando el padre exponía un argumento de calidad, el argumento del niño típicamente era un argumento de calidad. De manera similar, cuando el padre presentó un argumento de cantidad, el argumento del niño fue un argumento de cantidad. Debido a que durante las comidas los argumentos de calidad y cantidad son en la mayoría de los casos utilizados por padres e hijos para resaltar una propiedad específica (positiva o negativa) de los alimentos, estos dos argumentos, pueden definirse como vinculados al contexto. En línea con estudios precedentes (Brumark, 2008; Arcidiacono, Pontecorvo \& Greco Morasso, 2009), en el corpus la capacidad de los niños para justificar un punto de vista y utlizar una discusión con sus padres parece ser en gran parte dependiente del contexto.

Además, cabe destacar que cuando los padres usan argumentos de calidad y cantidad, a menudo adaptan su lenguaje al nivel de comprensión del niño. La elección de los padres de utilizar un nivel de lenguaje que los niños puedan comprender fácilmente es un rasgo típico de las interacciones argumentativas entre padres e hijos durante las comidas. Por ejemplo, si el propósito de los padres es alimentar a su hijo, 
la comida se describe como muy rica o nutritiva, y su cantidad es muy poca. Por el contrario, si el objetivo de los padres no es alimentar al niño más, en términos de calidad la comida se describe como salada o no rica, y en términos cuantitativos como es suficiente o es demasiado.

Los otros tipos de argumentos presentados por los padres, es decir, los argumentos de la autoridad, la apelación a la coherencia y el argumento de la analogía, aparecen con menos frecuencia en el corpus. Curiosamente, cuando los padres presentaron este tipo de argumentos, los niños no presentaron ningún argumento. Solo en dos casos, en reacción a un argumento de la autoridad, respondieron adelantando un argumento de la autoridad también. Por el contrario, su respuesta fue una expresión de otras dudas, por ejemplo, por qué-pregunta, o una mera oposición sin proporcionar ningún argumento en apoyo de su propia posición, por ejemplo, no, yo no quiero. En comparación con los argumentos de calidad y cantidad, el argumento de la autoridad, el pedido a la coherencia y el argumento de analogía utilizado por los padres parecen ser más complejos y elaborados. Lo interesante de este tipo de argumentos es el hecho de que introducen nuevos elementos en las interacciones entre padres e hijos, que no solo se relacionan con la evaluación de la calidad o cantidad de alimentos, sino que también abordan otros aspectos importantes que caracterizan las interacciones familiares. Nos referemos en particular a la enseñanza del comportamiento correcto en situaciones sociales dentro y fuera del contexto familiar, por ejemplo, en el contexto escolar con profesores y compañeros. Por esta razón, sostenemos que dentro de la actividad de la comida estos tipos de argumentos se pueden definir como sin contexto y, en nuestra opinión, esta puede ser una de las razones por las cuales los niños apenas presentan ningún argumento en reacción a estos tipos de argumentos presentados por sus padres. Además, dentro de este tipo de discusión, no hay diferencias entre los argumentos utilizados por las madres y los padres en nuestro corpus, excepto por el argumento de la autoridad que usan los padres en la mayoría de los casos. Este hallazgo muestra que el rol de los padres no habla en favor del uso de tipos específicos de argumentos durante las interacciones durante la comida con los niños.

Las estrategias argumentativas observadas implican no solo competencias discursivas, sino también elementos psicológicos, como la persuasión, la capacidad de convencer al interlocutor argumento, compromiso con las normas y reglas (Alzate \& Eugenio, 2012). Mediante su participación recíproca en las discusiones argumentativas, los padres y los niños producen y transforman conjuntamente el orden social y sus posiciones dentro de los marcos familiares, a través del formato y la secuencia de las acciones y sus respuestas. La dinámica de estos participantes es evidente en la colisión manifiesta de las maniobras de poder (Tannen, 2007) y la resistencia en las secuencias argumentativas. $\mathrm{Al}$ involucrarse en discusiones argumentativas, los padres aceptan el compromiso de transmitir reglas, valores y 
comportamientos correctos a sus hijos. Al participar en discusiones argumentativas con sus padres, los niños pueden ser más conscientes de su rol activo dentro del contexto familiar (Arcidiacono, 2007). La reconstrucción argumentativa de cómo los miembros de la familia resuelven dialécticamente las diferencias de opinión es, por lo tanto, una forma útil de resaltar las elecciones, formas y dinámicas adoptadas por adultos y niños a la hora de comer.

En general, los resultados de este estudio indican que los tipos de respuestas de los niños están estrictamente conectados con el tipo de argumento previamente utlizado por sus padres. Este aspecto es particularmente relevante en términos de las capacidades de los niños para participar en intercambios argumentativos y reaccionar de manera racional en el enfrentamiento con los padres. Durante las interacciones a la hora de la comida, los niños pueden usar el discurso para adquirir / mostrar un reconocimiento completo de que son miembros de la familia. Además, las habilidades argumentativas son la base sobre la cual los niños pueden desarrollar su papel de argumentadores también fuera del contexto familiar. Como ya observaron Ochs y colegas (Ochs, 2006; Ochs \& Schieffelin, 2011) y Pontecorvo y colegas (Pontecorvo \& Arcidiacono, 2007, 2010, 2014, 2016; Pontecorvo \& Pirchio, 2000), la importancia es examinar estas habilidades considerando las diferentes actividades diarias relevantes para el desarrollo del niño, como el contexto escolar durante las interacciones con otros adultos y compañeros, para iluminar otras áreas relevantes de la dinámica argumentativa adulto-niño (por ejemplo, Migdalek, Santibáñez Yáñez \& Rosemberg, 2014b; Migdalek, Rosemberg \& Arrúe, 2015). Centrándose en la interacción, la argumentación puede combinar el desarrollo constructivista con análisis discursivos y psicológicos cercanos: el método de análisis adoptado en este trabajo ha permitido un estudio detallado de las secuencias discursivas entre padres e hijos en una interacción multipartidista. Se necesita investigación adicional en esta dirección para comprender mejor las potencialidades específicas del lenguaje en el proceso cotidiano de socialización dentro del contexto familiar.

\section{REFERENCIAS BIBLIOGRÁFICAS}

Alam, F. \& Rosemberg, C. R. (2014). Narración y disputas entre niños. Un análisis de argumentaciones tempranas. Cogency, 6(1), 9-31.

Alzate, T. \& Eugenio, O. (2012). La argumentación como constituyente del pensamiento crítico en niños. Hallazgos, 9(17), 211-233.

Anderson, R. C., Chinn, C., Chang, J., Waggoner, M. \& Yi, M. (1997). On the logical integrity of children's arguments. Cognition and Instruction, 15(2), 135-167.

Arcidiacono, F. (2007). Conflitti e interazione in famiglia. Roma: Carocci. 
Arcidiacono, F. \& Pontecorvo, C. (2009). Cultural practices in Italian family conversations: Verbal conflict between parents and preadolescents. European Journal of Psychology of Education, 24(1), 97-117.

Arcidiacono, F. \& Bova, A. (2011). Argumentative strategies for conflict management and resolution in Italian and Swiss families. Procedia Social and Behavioral Sciences, 30, 1385-1389.

Arcidiacono, F. \& Bova, A. (2015). Activity-bound and activity-unbound arguments in response to parental eat-directives at mealtimes: Differences and similarities in children of 3-5 and 6-9 years old. Learning, Culture and Social Interaction, 6, 4055.

Arcidiacono, F. \& Bova, A. (2017). Interpersonal Argumentation in Educational and Professional Contexts. Nueva York: Springer.

Arcidiacono, F. \& Pontecorvo, C. (2009). Cultural practices in Italian family conversations: Verbal conflict between parents and preadolescents. European Journal of Psychology of Education, 24(1), 97-117.

Arcidiacono, F., Pontecorvo, C. \& Greco Morasso, S. (2009). Family conversations: The relevance of context in evaluating argumentation. Studies in Communications Sciences, 9(2), 79-92.

Bova, A. (2015a). This is the cheese bought by Grandpa. A study of the arguments from authority used by parents with their children during mealtimes. Journal of Argumentation in Context, 4(2), 133-157.

Bova, A. (2015b). Adult as a source of expert opinion in child's argumentation during family mealtime conversations. Journal of Argumentation in Context, 4(1), 4-20.

Bova, A. \& Arcidiacono, F. (2013a). Invoking the authority of feelings as a strategic maneuver in family mealtime conversations. Journal of Community and Applied Social Psychology, 23(3), 206-224.

Bova, A. \& Arcidiacono, F. (2013b). Investigating children's Why-questions. A study comparing argumentative and explanatory function. Discourse Studies, 15(6), 713-734.

Bova, A. \& Arcidiacono, F. (2014a). You must eat the salad because it is nutritious. Argumentative strategies adopted by parents and children in food-related discussions at mealtimes. Appetite, 73, 81-94.

Bova, A. \& Arcidiacono, F. (2014b). Types of arguments in parents-children discussions: An argumentative analysis. Rivista di Psicolinguistica Applicata/Journal of Applied Psycholinguistics, 14(1), 43-66. 
Bova, A. \& Arcidiacono, F. (2015). Beyond conflicts. Origin and types of issues leading to argumentative discussions during family mealtimes. Journal of Language Aggression and Conflict, 3(2), 263-288.

Bova, A. \& Arcidiacono, F. (2018). Interplay between parental argumentative strategies, children's reactions, and topics of disagreement during mealtime conversations. Learning, Culture and Social Interaction, 19, 124-133.

Blum-Kulka, S. (1997). Dinner talk: Cultural patterns of sociability and socialization in family discourse. Mahwah, NJ: Erlbaum.

Brumark, Å. (2008). Eat your hamburger! - No, I don't want to! Argumentation and argumentative development in the context of dinner conversation in twenty Swedish families. Argumentation, 22(2), 251-271.

Dunn, J. \& Munn, P. (1987). Developmental of justification in disputes with mother and sibling. Developmental Psychology, 23(6), 791-798.

Fiese, B. H., Foley, K. P. \& Spagnola, M. (2006). Routine and ritual elements in family mealtimes: Contexts for child well-being and family identity. New Directions for Child and Adolescent Development, 111, 67-89.

Goetz, J. P. (2010). The development of verbal justifications in the conversations of preschool children and adults. First Language, 30(3), 403-420.

Goodwin, C. (2006). Retrospective and prospective orientation in the construction of argumentative moves. Text and Talk, 26(4-5), 443-461.

Goodwin, M. H. (2006). Participation, affect, and trajectory in family directive/response sequences. Text \& Talk, 26(4-5), 515-543.

Hester, S. \& Hester, S. (2010). Conversational actions and category relations: An analysis of a children's argument. Discourse Studies, 12(1), 33-48.

MacWhinney, B. (2000). The CHILDES Project: Tools for Analyzing Talk. 3rd Edition. Mahwah, NJ: Erlbaum.

Migdalek, M. J., Rosemberg, C. R. \& Santibáñez Yáñez, C. (2014a). La génesis de la argumentación. Un estudio con niños de 3 a 5 años en distintos contextos de juego. Ikala, Revista de Lenguaje y Cultura, 19(3), 251-267.

Migdalek, M. J., Santibáñez Yáñez, C. \& Rosemberg, C. R. (2014b). Estrategias argumentativas en niños pequeños: Un estudio a partir de las disputas durante el juego en contextos escolares. Revista Signos. Estudios de Lingüística, 47(86), 435-462. 
Migdalek, M. J., Rosemberg, C. R. \& Arrúe, J. E. (2015). Argumentación infantil en situaciones de juego: Diferencias en función del contexto. Propuesta Educativa, 24(44), 79-88.

Muller Mirza, N. \& Perret-Clermont, A.-N. (2009). Argumentation and education. theoretical foundations and practices. Nueva York, NY: Springer.

Ochs, E. (2006). The cultural structuring of mealtime socialization. New Directions for Child and Adolescent Development, 111, 35-49.

Ochs, E. \& Schieffelin, B. B. (2011). The theory of language socialization. En A. Duranti, E. Ochs \& B. B. Schieffelin (Eds.), The Handbook of Language Socialization (pp. 1-21). Malden, MA: Wiley-Blackwell.

Ochs, E., Pontecorvo, C. \& Fasulo, A. (1996). Socializing taste. Ethnos, 61(1-2), 7-46.

Orsolini, M. (1993). Dwarfs do not shoot: An analysis of children's justifications. Cognition and Instruction, 11(3-4), 281-297.

Pauletto, F., Aronsson, K. \& Arcidiacono, F. (2017). Intergenerational argumentation: Children's account work during dinner conversations in Italy and Sweden. En F. Arcidiacono \& A. Bova (Eds.), Interpersonal Argumentation in Educational and Professional Contexts (pp. 1-26). Nueva York, NY: Springer.

Pontecorvo, C. \& Fasulo, A. (1999). Planning a typical Italian meal: A family reflection on culture. Culture \& Psychology, 5(3), 313-335.

Pontecorvo, C. \& Pirchio, S. (2000). A developmental view on children's arguing: The need of the other. Human Development, 43(6), 361-363.

Pontecorvo, C. \& Arcidiacono, F. (2007). Famiglie all'italiana. Parlare a tavola. Milán: Cortina.

Pontecorvo, C. \& Arcidiacono, F. (2010). Development of reasoning through arguing in young children. Культурно-Историческая Психология / Cultural-Historical Psychology, 4, 19-30.

Pontecorvo, C. \& Arcidiacono, F. (2014). Social interactions in families and schools as contexts for the development of spaces of thinking. En T. Zittoun \& A. Iannaccone (Eds.), Activity of thinking in social spaces (pp. 83-97). Nueva York: Nova Science Publishers.

Pontecorvo, C. \& Arcidiacono, F. (2016). The dialogic construction of justifications and arguments in a seven-year-old child within a democratic family. Language and Dialogue, 6(2), 306-328. 
Pontecorvo, C., Fasulo, A. \& Sterponi, L. (2001). Mutual apprentices: Making of parenthood and childhood in family dinner conversations. Human Development, 44(6), 340-361.

Schiffrin, D. (1987). Discourse markers. Cambridge: Cambridge University Press.

Stein, N. L. \& Albro, E. R. (2001). The origins and nature of arguments: Studies in conflict understanding, emotion and negotiation. Discourse Processes, 32(2-3), 113-133.

Tannen, D. (2007). Power maneuvers and connection maneuvers in family interaction. En D. Tannen, S. Kendall \& C. Gordon (Eds.), Discourse and Identity in Four American Families (pp. 27-48). Nueva York: Oxford University Press.

Van Eemeren, F. H. \& Grootendorst, R. (1992). Argumentation, communication, and fallacies. A pragma-dialectical perspective. Hillsdale, NJ: Erlbaum.

Van Eemeren, F. H. \& Grootendorst, R. (2004). A systematic theory of argumentation: The pragma-dialectical approach. Cambridge: Cambridge University Press.

Walton, D. N. (1997). Appeal to expert opinion. Arguments from authority. Pennsylvania: State University Press.

Walton, D. N., Reed, C. \& Macagno, F. (2008). Argumentation Schemes. Nueva York: Cambridge University Press.

\section{NOTA}

1 Basado en las respuestas de los padres a los cuestionarios sobre el estado socioeconómico (SES) y los datos personales de los familiares que los participantes llenaron antes de las grabaciones de video. 